Claremont Colleges

Scholarship@ Claremont

All HMC Faculty Publications and Research

HMC Faculty Scholarship

6-1-2011

\title{
Existence of Solutions for a Wave Equation with Non-monotone Nonlinearity and a Small Parameter
}

Jose F. Caicedo

Universidad Nacional de Colombia

Alfonso Castro

Harvey Mudd College

Rodrigo Duque

Universidad Nacional de Colombia

\section{Recommended Citation}

Caicedo, Jose F., Alfonso Castro and Rodrigo Duque. "Existence of Solutions for a Wave Equation with Non-monotone Nonlinearity and a Small Parameter", Milan Journal of Mathematics, Volume 79, Number 1, (2011), pp. 207-220.

This Article - postprint is brought to you for free and open access by the HMC Faculty Scholarship at Scholarship @ Claremont. It has been accepted for inclusion in All HMC Faculty Publications and Research by an authorized administrator of Scholarship @ Claremont. For more information, please contact scholarship@cuc.claremont.edu. 


\title{
Existence of Solutions for a Wave Equation with Non-monotone Nonlinearity and a Small Parameter
}

\author{
José F. Caicedo, Alfonso Castro and Rodrigo Duque*
}

\begin{abstract}
We provide sufficient conditions for the existence of solutions to a semilinear wave equation with non-monotone nonlinearity involving a small parameter. Our results are based on the analysis of a an operator that characterizes the projection onto the kernel of the wave operator subject to periodic-Dirichlet boundary conditions. Such a kernel is infinite dimensional which makes standard compactness arguments inapplicable.
\end{abstract}

Mathematics Subject Classification (2010). Primary 35L75; Secondary 34B15.

Keywords. Semilinear wave equation, characteristic line, infinite dimensional kernel.

\section{Introduction}

Based on the results of [1] and the methods introduced in [6], we study the equation

$$
\left\{\begin{array}{l}
\square u=\epsilon\left(u^{2 k}+h(t, x)+R(t, x, u)\right) \\
u(t, 0)=u(t, \pi)=0 \\
u(t, x)=u(t+2 \pi, x)
\end{array}\right.
$$

where $\square=\partial_{t t}-\partial_{x x}$ denotes the D'Alembert operator, $k$ is a positive integer, $t \in \mathbb{R}, x \in[0, \pi]$ and $R \in C^{0}(\mathbb{R} \times[0, \pi] \times \mathbb{R})$ is $2 \pi$-periodic in its first variable. We assume that $R$ is differentiable in its third variable, and that

$$
R(t, x, 0)=0 \text { and } \lim _{u \rightarrow 0} \frac{R_{u}(t, x, u)}{u^{2 k-1}}=0,
$$

uniformly for $(t, x) \in \mathbb{R} \times[0, \pi]$.

The key feature of equation (1) is that, regardless of the size of $\epsilon$, the derivative of the nonlinearity includes the eigenvalue 0 which has infinite multiplicity (see (3) below) making compactness arguments not applicable. All the results of this paper

\footnotetext{
*Part of this research was done while the third author was a UNIALA doctoral fellow.
} 
extend to the case where, in (1), $u^{2 k}$ is replaced by $\beta(x) u^{2 k}$ with $\beta$ a positive continuous function such $\beta(x)=\beta(\pi-x)$. For the sake of simplicity in the presentation we restrict ourselves to the case $\beta(x) \equiv 1$.

Throughout this paper all functions are $2 \pi$-periodic in the variable $t$.

The kernel of $\square$ subject to the boundary conditions in (1) is

$$
\begin{aligned}
N & =\left\{v(t, x)=p(t+x)-p(t-x) ; p \in L^{2}([0,2 \pi]), \int_{0}^{2 \pi} p(s) d s=0\right\} \\
& =\left\{v(t, x)=p(t+x)-p(t-x) ; p \in L^{2}([0,2 \pi])\right\} .
\end{aligned}
$$

Let $\Omega=[0,2 \pi] \times[0, \pi]$,

$$
\begin{aligned}
& N^{\perp}=\left\{w: \mathbb{R} \times[0, \pi] \rightarrow \mathbb{R} ; w \in L^{2}(\Omega), \int_{\Omega} w v=0 \quad \forall v \in N\right\}, \\
& \mathbf{H}^{\mathbf{1}}=\left\{w: \mathbb{R} \times[0, \pi] \rightarrow \mathbb{R} ; w, w_{t}, w_{x} \in L^{2}(\Omega), w(t, 0)=w(t, \pi)=0\right\},
\end{aligned}
$$

For $1 \leq p<\infty$, the norm in $L^{p}(\Omega)$ will be denoted by $\|\cdot\|_{p}$; the norms in $L^{\infty}$ and $C^{0}$ will simply be denoted by $\|\cdot\|$. The norm in the space $\mathbf{H}^{\mathbf{1}}$ will be denoted by $\|\cdot\|_{1,2}$ and is defined as

$$
\|w\|_{1,2}=\left(\int_{\Omega}\left(w_{t}^{2}(t, x)+w_{x}^{2}(t, x)\right) d t d x\right)^{1 / 2} .
$$

An elementary argument based on Fourier expansions shows that for each $f \in N^{\perp}$ there exists a unique $w \in \mathbf{H}^{\mathbf{1}} \cap N^{\perp}$ such that $\square(w)=f$ in the sense of distributions. Moreover, the transformation $f \rightarrow w \equiv \square^{-1}(f)$ is continuous as an operator from $L^{2}(\Omega) \cap N^{\perp}$ into $\mathbf{H}^{\mathbf{1}} \cap N^{\perp}$, from $L^{2}(\Omega) \cap N^{\perp}$ into $L^{\infty} \cap N^{\perp}$, and from $L^{\infty}(\Omega) \cap N^{\perp}$ into $C^{0,1} \cap N^{\perp}$. Thus there exists a constant $c_{0}$ such that

$$
\left\|\square^{-1} f\right\|_{1,2} \leq c_{0}\|f\|_{2},\left\|\square^{-1} f\right\|_{\infty} \leq c_{0}\|f\|_{2} \text {, and }\left\|\square^{-1} f\right\|_{C^{0,1}} \leq c_{0}\|f\|_{\infty},
$$

see [1], (2.3).

For the rest of this paper $h \in N^{\perp}$. Letting $H=\square^{-1}(h)+v$, with $v \in N$, subtracting $\square(\epsilon H)=\epsilon h$ from both sides of the first equation in (1) and replacing $u-\epsilon H$ by $u$ that equation becomes

$$
\square u=\epsilon(u+\epsilon H)^{2 k}+\epsilon R(t, x, u+\epsilon H),
$$

subject to the the boundary conditions in (1).

We establish the solvability of $(7)$ in terms of the operator $L_{J}: C^{0}((0,2 \pi)) \rightarrow$ $C^{0}((0,2 \pi))$ defined by

$$
\begin{aligned}
\left(L_{J}(p)\right)(r)= & p(r) \int_{0}^{\pi}\{J(r+x, x)+J(r-x, x)\} d x \\
& -\int_{0}^{\pi}\{p(r+2 x) J(r+x, x)+p(r-2 x) J(r-x, x)\} d x
\end{aligned}
$$

where $p(t+x)-p(t-x)=v(t, x) \in N, \quad r \in[0,2 \pi]$.

In fact, our main result is: 
Theorem 1. Suppose $(2)$ is satisfied and let $\Pi_{N}$ denote the $L^{2}(\Omega)$-orthogonal projection onto $N$. If

a) $k=1$ and $L_{H}$ is invertible, or

b) $k>1$, and there exists $\hat{v} \in N$ such that $\|\hat{v}\| \leq O(\epsilon), \Pi_{N}(\hat{v}+\epsilon H)^{2 k}=0$ and $L_{J}$ is invertible for $J=(\hat{v}+\epsilon H)^{2 k-1}-\hat{v}^{2 k-1}$ with $\epsilon^{1-2 k}\left\|L_{J}\right\|$ bounded away from zero,

then there exist $\epsilon_{0}>0$ such that for $\epsilon \in\left(0, \epsilon_{0}\right)$ the equation (7) has a solution $u \in C^{0}(\Omega)$.

Our next result shows that Theorem 1 includes the existence results in [1]. In fact, we have:

Theorem 2. If, for some $v \in N, H$ is continuous and $H(t, x)>0$ for all $(t, x) \in \Omega$, then $L_{H}$ is invertible. If, in addition, $k>1$ then there exists $\hat{v} \in N$ satisfying part b) in Theorem 1. Hence there exist $\epsilon_{0}>0$ such that for $\epsilon \in\left(0, \epsilon_{0}\right)$ the equation (7) has a solution $u \in C^{0}(\Omega)$.

The positivity of $H$ is not a necessary condition for the invertivility of $L_{H}$. For example if $h(t, x)=9 \sin (3 x)+h_{1}(t, x)$ with $h_{1}$ small enough then $H=\square^{-1} h+v$ changes sign for any $v \in N$ and yet $L_{H}$ is invertible. This is a case where Theorem 1 applies but not the results of [1]. See Theorem 3 in Section 5 below.

The operator $L_{H}$ was introduced in [5] to prove the nonexistence of continuous solutions to

$$
\square u=g(u)+\lambda u+h(t, x), \quad u(t, x)=u(t+2 \pi, x)=u(t, x+2 \pi)
$$

when $\lambda$ is not an eigenvalue of $\square$ subject to the periodicity condition in (9), g has compact support, $\lambda u+g(u)$ is not monotone, and $h$ is a large multiple of $\sin (t+x)$. The operator $L_{H}$ was also used in [6] to prove the existence of solutions to (9) when $h$ does not vanish on sets of positive measure in any characteristic line. For results on $(9)$ the reader is referred to $[2,3,12]$, for the other studies on the non-monotone case see $[4,9,13]$.

Following the results in [8], if the functions $h$ and $R$ satisfy the symmetry $h(t, x)=h(t+\pi, \pi-x), R(t, x, u)=R(t+\pi, \pi-x, u)$ then one may restrict the study of equation (1) to spaces of functions $u$ with this symmetry. Since no non-zero element in $N$ satisfies this symmetry, $\Pi_{N}\left((u+\epsilon H)^{2 k}+R(t, x, u+\epsilon H)\right)=0$ for any $u$ satisfying the symmetry (see (10) below). This reduces the solvability of (1) to the solvability of the range equation (11) which is easily solved for $\epsilon$ small under no additional hypothesis on $h$, see Section 2 .

The solutions given by Theorem 1 satisfy $\|u\| \leq O(\epsilon)$. This cannot happen if $h \notin N^{\perp}$, see [1], Remark 1.1. Thus the assumption $h \in N^{\perp}$ is necessary. If $H>0$ and smooth the solutions to (1) are smooth (see [1], Theorem 2.) The regularity of the solutions here obtained for $H$ changing sign is yet to be studied. 


\section{Solvability in $N^{\perp}$}

Let $V=N \cap C^{0}(\Omega), W=N^{\perp} \cap C^{0}(\Omega)$ and $\Pi_{N}, \Pi_{N^{\perp}}$ the orthogonal projections from $L^{2}(\Omega)$ onto $N$ and $N^{\perp}$, respectively.

Setting $u=v+w$ with $v \in V$ and $w \in W$, the problem (7) is equivalent to solving the kernel and the range equations

$$
\begin{gathered}
\Pi_{N}\left((v+w+\epsilon H)^{2 k}+R(t, x, v+w+\epsilon H)\right)=0, \\
\epsilon \square^{-1} \Pi_{N^{\perp}}\left((v+w+\epsilon H)^{2 k}+R(t, x, v+w+\epsilon H)\right)=w .
\end{gathered}
$$

In order to solve the kernel equation, (10), we will follow the methods introduced in $[5,6]$. The following proposition summarizes the solvability of the range equation, (11). We omit its proof as, up to minor details, it is given by the proof of Proposition 3.2 of $[1]$.

Proposition 1. There exist $\hat{\epsilon}>0$ and $\delta_{0}>0$ such that if $v \in V$ with $\|v\|_{C^{0}} \leq \delta_{0}$ and $|\epsilon|<\hat{\epsilon}$ then (11) has a unique solution $w(v, \epsilon) \in N^{\perp}$. Moreover there exists $\alpha>0$ such that

$$
\|w(v, \epsilon)\| \leq \alpha|\epsilon|\left(\|v\|^{2 k}+|\epsilon|^{2 k}\right) \text { and }\left\|w\left(v_{1}, \epsilon\right)-w\left(v_{2}, \epsilon\right)\right\| \leq|\epsilon| \alpha\left\|v_{1}-v_{2}\right\|,
$$

for all $v, v_{1}, v_{2}$ with $\|v\|,\left\|v_{1}\right\|,\left\|v_{2}\right\| \leq \delta_{0}$, and $|\epsilon| \leq \hat{\epsilon}$.

\section{Proof of Theorem 1}

We prove in detail the case $k>1$; the case $k=1$ follows the same pattern with $\hat{v}=0$ making the calculations a lot simpler.

Let $\hat{v}$ be as in part b) of Theorem 1 , and $v=\hat{v}+\zeta$. Since the product of an even number of elements in $N$ is in $N^{\perp}, \Pi_{N}\left(\hat{v}^{2 k-1} \zeta\right)=0$ (see [1, Lemma 2.4]). Therefore (10) is equivalent to

$$
\begin{aligned}
0= & \Pi_{N}\left(-2 k \hat{v}^{2 k-1} \zeta+(\hat{v}+\epsilon H)^{2 k}+2 k(\hat{v}+\epsilon H)^{2 k-1} \zeta+2 k(\hat{v}+\epsilon H)^{2 k-1} w\right. \\
& \left.+\sum_{j=2}^{2 k} C_{j}(\hat{v}+\epsilon H)^{2 k-j}(\zeta+w)^{j}+R(t, x, \hat{v}+\zeta+w+\epsilon H)\right) \\
= & \Pi_{N}\left(2 k J \zeta+2 k(\hat{v}+\epsilon H)^{2 k-1} w+\sum_{j=2}^{2 k} C_{j}(\hat{v}+\epsilon H)^{2 k-j}(\zeta+w)^{j}\right. \\
& +R(t, x, \hat{v}+\zeta+w+\epsilon H)) \\
\equiv & \Pi_{N}(2 k J \zeta+Q(\zeta, \epsilon, H)),
\end{aligned}
$$

where $C_{j}$ is the binomial coefficient $2 k$ choose $j$, and $J=(\hat{v}+\epsilon H)^{2 k-1}-\hat{v}^{2 k-1}$.

Let $z: \mathbb{R} \rightarrow \mathbb{R}$ be a $2 \pi$-periodic function such that $\zeta(t, x)=z(t+x)-z(t-x)$ with $\int_{0}^{2 \pi} z(s) d s=0$. 
Vol. 79 (2011)

As in [6], for $0 \leq r \leq s \leq 2 \pi$ let $\chi_{[r, s]}$ be the $2 \pi$-periodic function such that

$$
\chi_{[r, s]}(t)= \begin{cases}1, & t \in[r, s] \\ 0, & t \in[0,2 \pi]-[r, s]\end{cases}
$$

and let $\phi$ be the function defined by

$$
\phi(t, x)=\chi_{[r, s]}(t+x)-\chi_{[r, s]}(t-x) \in N
$$

Also we let

$$
\begin{aligned}
& A=\left\{(t, x) \in \Omega ; x \in[0, \pi], t \in \bigcup_{j}[r+2 j \pi-x, s+2 j \pi-x],\right. \\
& \quad j=0,1\} \\
& B=\left\{(t, x) \in \Omega ; x \in[0, \pi], t \in \bigcup_{j}[r+2 j \pi+x, s+2 j \pi+x],\right. \\
& \quad j=-1,0\} .
\end{aligned}
$$

From now on, for the sake of simplicity in the notations, we write $d t d x=d \sigma$.

Multiplying $\zeta J$ by $\phi$ and integrating on $\Omega$ we obtain

$$
\begin{aligned}
\int_{\Omega} \zeta J \phi d \sigma= & \int_{\Omega} z(t+x) J(t, x) \phi(t, x) d \sigma-\int_{\Omega} z(t-x) J(t, x) \phi(t, x) d \sigma \\
= & \int_{B} z(t+x) J(t, x) d \sigma-\int_{A} z(t+x) J(t, x) d \sigma \\
& -\int_{B} z(t-x) J(t, x) d \sigma+\int_{A} z(t-x) J(t, x) d \sigma \\
= & \int_{0}^{\pi} \int_{r}^{s}(z(\eta) J(\eta-x, x)-z(\eta+2 x) J(\eta+x, x)) d \eta d x \\
& -\int_{0}^{\pi} \int_{r}^{s}(z(\eta-2 x) J(\eta-x, x)-z(\eta) J(\eta+x, x)) d \eta d x .
\end{aligned}
$$

By the Lebesgue differentiation theorem (see (49) in [2]), for almost every $r \in[0,2 \pi]$,

$$
\begin{aligned}
\lim _{s \rightarrow r} \frac{1}{s-r} \int_{\Omega} \zeta J \phi d \sigma= & \int_{0}^{\pi}(z(r)-z(r-2 x)) J(r-x, x) d x \\
& +\int_{0}^{\pi}(z(r)-z(r+2 x)) J(r+x, x) d x \\
= & {\left[L_{J}(z)\right](r) . }
\end{aligned}
$$

Similarly, multiplying $Q(\zeta, \epsilon, H)$ by $\phi$, integrating on $\Omega$, dividing by $s-r$, and taking limit as $s$ tend to $r$ we have

$$
\begin{aligned}
& \lim _{s \rightarrow r} \frac{1}{s-r} \int_{\Omega} Q(\zeta, \epsilon, H) \phi d \sigma= \int_{0}^{\pi} Q(\zeta, \epsilon, H)(r-x, x) d x \\
&\left.+\int_{0}^{\pi} Q(\zeta, \epsilon, H)(r+x, x)\right) d x \\
& \equiv[\Gamma(z)](r) .
\end{aligned}
$$


Hence if $v=\hat{v}+\zeta$ and $w=w(v, \epsilon)$ satisfies (10) then $2 k L_{J}(z)=\Gamma(z)$ (see Proposition $1)$. Conversely, if $z$ satisfies $z=(1 /(2 k)) L_{J}^{-1} \Gamma(z) \equiv \Gamma_{1}(z)$ then $v+w(v, \epsilon)$ satisfies (10) (see [6, Lemma 1.3].) Therefore $v+w(v, \epsilon)$ solves (1).

Let $M>0$ be such that $\|\hat{v}\|+\epsilon\|H\| \leq M \epsilon$ for all $\epsilon \in(0, \hat{\epsilon})$. Due to assumption $b)$, by further restricting $\hat{\epsilon}$ if needed, there exists $m>0$ such that $\epsilon^{2 k-1}\left\|L^{-1}\right\| \leq m$ for all $\epsilon \in(0, \hat{\epsilon})$. Since $k>1$, there exist $\epsilon_{3} \in(0, \hat{\epsilon})$ and $\tau>0$ be such that

$$
\begin{aligned}
& 2 k M^{2 k-1} \alpha \mu \epsilon_{3}^{2 k}+\sum_{j=2}^{2 k} D_{j}\left(2 \tau+\alpha \mu \epsilon_{3}^{2 k}\right)^{j} \leq \frac{\tau}{4 \pi}, \\
& m\left(2 k \alpha M^{2 k-1} \epsilon_{3}+\sum_{j=2}^{2 k} j D_{j}\left(2 \tau+\alpha \epsilon_{3}^{2 k} \mu\right)^{j-1}\right) \leq \frac{1}{8 \pi},
\end{aligned}
$$

where $D_{j}=C_{j} M^{2 k-j}$ and $\mu=(M+2 \tau)^{2 k}+1$. Next we choose $\gamma>0$ be such that

$$
4 \pi \gamma<\min \left\{\frac{\tau}{\left(M+2 \tau+\alpha \epsilon_{3}^{2 k} \mu\right)^{2 k}}, \frac{1}{2 m\left(1+\alpha \epsilon_{3}\right)\left(2 \tau+M+\alpha \mu \epsilon_{3}^{2 k}\right)^{2 k-1}}\right\} .
$$

By (2), there exists $\delta>0$ such that if $|s|<\delta$ then $|R(t, x, s)|<\gamma s^{2 k}$ and $\left|R_{u}(t, x, s)\right| \leq \gamma|s|^{2 k-1}$. Finally we take $\epsilon_{4} \in\left(0, \epsilon_{3}\right)$ such that

$$
\epsilon_{4}\left(2 M+2 \tau+\alpha \epsilon_{4}^{2 k}\right)<\delta
$$

Now for $\|z\| \leq \tau \epsilon$ we have $\|\zeta\| \leq 2 \tau \epsilon$, and $\|w(\hat{v}+\zeta, \epsilon)\| \leq \alpha(M+2 \tau)^{2 k} \epsilon^{2 k+1}$. Therefore

$$
\begin{aligned}
\|Q(\zeta, \epsilon, H)\| \leq & 2 k\left\|(\hat{v}+\epsilon H)^{2 k-1} w\right\|+\sum_{j=2}^{2 k} C_{j}(M \epsilon)^{2 k-j}\|\zeta+w\|^{j} \\
& +\|R(t, x, \hat{v}+\zeta+w+\epsilon H)\| \\
\leq & 2 k M^{2 k-1} \alpha(M+2 \tau)^{2 k} \epsilon^{4 k} \\
& +\sum_{j=2}^{2 k} D_{j} \epsilon^{2 k-j}\left(2 \tau \epsilon+\alpha(M+2 \tau)^{2 k} \epsilon^{2 k+1}\right)^{j} \\
& +\gamma \| \hat{v}+\zeta+w+\epsilon H) \|^{2 k} \\
\leq & \epsilon^{2 k}\left(2 k M^{2 k-1} \alpha(M+2 \tau)^{2 k} \epsilon^{2 k}\right. \\
& \sum_{j=2}^{2 k} D_{j}\left(2 \tau+\alpha(M+2 \tau)^{2 k} \epsilon^{2 k}\right)^{j} \\
& \left.+\gamma\left(M+2 \tau+\alpha \mu \epsilon^{2 k}\right)\right) \\
\leq & \tau \epsilon^{2 k}
\end{aligned}
$$


Let $\zeta_{i}(t, x)=z_{i}(t+x)-z_{i}(t-x)$ for $i=1,2$, and $w_{i}=w\left(\hat{v}+\zeta_{i}, \epsilon\right)$ with $\left\|z_{i}\right\| \leq \tau \epsilon$.

Thus, from the definition of $Q$, Proposition 1, (20), (21) and (22), we have

$$
\begin{aligned}
\| Q\left(\zeta_{1}, \epsilon,\right. & H)-Q\left(\zeta_{2}, \epsilon, H\right)\|\leq 2 k\|(\hat{v}+\epsilon H)^{2 k-1}\|\| w_{1}-w_{2} \| \\
& +\sum_{j=2}^{2 k} C_{j}(M \epsilon)^{2 k-j}\left\|\left(\zeta_{1}+w_{1}\right)^{j}-\left(\zeta_{2}+w_{2}\right)^{j}\right\| \\
& +\left\|R\left(t, x, \hat{v}+\zeta_{1}+w_{1}+\epsilon H\right)-R\left(t, x, \hat{v}+\zeta_{2}+w_{2}+\epsilon H\right)\right\| \\
\leq & 2 k(M \epsilon)^{2 k-1} \alpha \epsilon\left\|\zeta_{1}-\zeta_{2}\right\| \\
& +\left(\sum_{j=2}^{2 k} D_{j} \epsilon^{2 k-j}(1+\epsilon \alpha) \sum_{i=0}^{j-1}\left\|\left(\zeta_{1}+w_{1}\right)^{j-1-i}\left(\zeta_{2}+w_{2}\right)^{i}\right\|\right. \\
& \left.+\gamma(2 M+1+\alpha)^{2 k-1} \epsilon^{2 k-1}\right)\left\|\zeta_{1}-\zeta_{2}\right\| \\
\leq & \epsilon^{2 k-1}\left(\sum_{j=2}^{2 k} D_{j}(1+\epsilon \alpha) j\left(2 \tau+\alpha \mu \epsilon^{2 k}\right)^{j-1}\right. \\
& \left.+2 k M^{2 k-1} \alpha \epsilon+\gamma(1+\alpha \epsilon)\left(2 \tau+M+\alpha \mu \epsilon^{2 k}\right)^{2 k-1}\right)\left\|\zeta_{1}-\zeta_{2}\right\| \\
\leq & \frac{1}{2\left\|L_{J}^{-1}\right\|}\left\|\zeta_{1}-\zeta_{2}\right\| .
\end{aligned}
$$

From $(23)$ we see that $\left(\epsilon^{1-2 k} /(2 k)\right) L_{J}^{-1} \Gamma$ transforms the metric space $\{z ;\|z\| \leq \tau \epsilon\}$ into itself. Also (24) proves that $\left(\epsilon^{1-2 k} /(2 k)\right) L_{J}^{-1} \Gamma$ is a contraction. Hence it has a unique fixed point which proves Theorem 1.

\section{Proof of Theorem 2}

Let

$$
X_{2}=\left\{p \in C^{0}(\mathbb{R}) ; p(x)=p(x+2 \pi), \int_{0}^{2 \pi} p(s) d s=0,\|p\|_{C^{0}}=1\right\}
$$

For each $p \in X_{2}$, let $r_{p} \in[0,2 \pi]$ be such that $\left|p\left(r_{p}\right)\right|=1$. We claim that

$$
\inf _{p \in X_{2}}\left|L_{H}(p)\left(r_{p}\right)\right|>0 .
$$

Let us assume that there exists a sequence $\left\{p_{n}\right\}$ in $X_{2}$ such that $\left|L_{H}\left(p_{n}\right)\left(r_{p_{n}}\right)\right|<1 / n$. Without loss of generality we may assume that $p_{n}\left(r_{p_{n}}\right)=1$. Hence

$$
\begin{aligned}
\frac{1}{n} \geq & L_{H}\left(p_{n}\right)\left(r_{p_{n}}\right) \\
= & \int_{0}^{\pi}\left(1-p_{n}\left(r_{p_{n}}-2 x\right)\right) H\left(r_{p_{n}}-x, x\right) d x \\
& +\int_{0}^{\pi}\left(1-p_{n}\left(r_{p_{n}}+2 x\right)\right) H\left(r_{p_{n}}+x, x\right) d x \\
\geq & \int_{0}^{\pi}\left(1-p_{n}\left(r_{p_{n}}-2 x\right)\right) H\left(r_{p_{n}}-x, x\right) d x .
\end{aligned}
$$


Therefore

$$
\left\{\sqrt{\left(1-p_{n}\left(r_{p_{n}}+2 x\right)\right) H\left(r_{p_{n}}+x, x\right)}\right\} \rightarrow 0 \text {, in } L^{2} .
$$

Hence there exists a subsequence $\left\{p_{n_{k}}\right\}$ of $\left\{p_{n}\right\}$ such that

$$
\left\{\left(1-p_{n_{k}}\left(r_{p_{n_{k}}}+2 x\right)\right) H\left(r_{p_{n_{k}}}+x, x\right)\right\} \rightarrow 0
$$

almost everywhere on $[0, \pi]$. Since $H(t, x)>0, \forall(t, x) \in \Omega$, then the sequence $\left\{p_{n_{k}}\right\} \rightarrow 1$ a.e. $[0,2 \pi]$. But this is a contradiction, because $\int_{0}^{2 \pi} p_{n_{k}}(s) d s=0$ for all $k$. Hence $(25)$ is proven, which proves that $L_{H}$ is invertible. Thus the first statement in Theorem 2 is proven.

In order to prove the second statement in Theorem 2 we define $\hat{v}=\epsilon V$, with $V$ as given by Lemma 3. Since $(\hat{v}+\epsilon H)^{2 k-1}-\hat{v}^{2 k-1} \geq 2((\epsilon H) / 2)^{2 k-1}$ the second stament in Theorem 2 is proven. The third statement in Theorem 2 follows from Theorem 1, which completes the proof of Theorem 2.

\section{Examples of invertivility of $L$ when $H$ changes sign}

In this section we make use of Fourier expansions to provide examples in which $L_{H} \equiv L$ (see (8), Theorem 1) is invertible in the space of continuous functions and yet $H$ changes sign. In fact we show that this is the case for $H(t, x)=\sin (3 x)$, and explicitely calculate $L^{-1}$. This and Theorem 2 prove that our results properly include those of [1].

Let

$$
\begin{aligned}
H(t, x) & =\sum_{j=1, l=0}^{\infty, \infty}\left(a_{j l} \sin (j x) \sin (l t)+b_{j l} \sin (j x) \cos (l t)\right), \\
p(t) & =\sum_{k=1}^{\infty}\left(c_{k} \sin (k t)+d_{k} \cos (k t)\right) .
\end{aligned}
$$

Elementary calculations show that

$$
\begin{aligned}
L(\sin (k r))= & 8 \sum_{j+l \text { odd }}\left(\frac{j a_{j l}\left(k^{2}-k l\right)}{\left(j^{2}-l^{2}\right)\left((2 k-l)^{2}-j^{2}\right)} \cos (k r-l r)\right. \\
& -\frac{j a_{j l}\left(k^{2}+k l\right)}{\left(j^{2}-l^{2}\right)\left((2 k+l)^{2}-j^{2}\right)} \cos (k r+l r) \\
& +\frac{j b_{j l}\left(k^{2}-k l\right)}{\left(j^{2}-l^{2}\right)\left((2 k-l)^{2}-j^{2}\right)} \sin (k r-l r) \\
& \left.+\frac{j b_{j l}\left(k^{2}+k l\right)}{\left(j^{2}-l^{2}\right)\left((2 k+l)^{2}-j^{2}\right)} \sin (k r+l r)\right) .
\end{aligned}
$$


Similarly

$$
\begin{aligned}
L(\cos (k r))= & 8 \sum_{j+l \text { odd }}\left(\frac{j b_{j l}\left(k^{2}-k l\right)}{\left(j^{2}-l^{2}\right)\left((2 k-l)^{2}-j^{2}\right)} \cos (k r-l r)\right. \\
& +\frac{j b_{j l}\left(k^{2}+k l\right)}{\left(j^{2}-l^{2}\right)\left((2 k+l)^{2}-j^{2}\right)} \cos (k r+l r) \\
& -\frac{j a_{j l}\left(k^{2}-k l\right)}{\left(j^{2}-l^{2}\right)\left((2 k-l)^{2}-j^{2}\right)} \sin (k r-l r) \\
& \left.+\frac{j a_{j l}\left(k^{2}+k l\right)}{\left(j^{2}-l^{2}\right)\left((2 k+l)^{2}-j^{2}\right)} \sin (k r+l r)\right) .
\end{aligned}
$$

In particular, if $H(t, x)=\sin (3 x)$

$$
L(\sin (k r))=\frac{16 k^{2}}{3\left(4 k^{2}-9\right)} \sin (k r)
$$

and

$$
L(\cos (k r))=\frac{16 k^{2}}{3\left(4 k^{2}-9\right)} \cos (k r) .
$$

Hence, for $H(t, x)=\sin (3 x)$

$$
\begin{aligned}
L(p(t)) & =L\left(\sum_{k=1}^{\infty}\left(c_{k} \sin (k t)+d_{k} \cos (k t)\right)\right) \\
& =\sum_{k=1}^{\infty} \frac{16 k^{2}}{3\left(4 k^{2}-9\right)}\left(c_{k} \sin (k t)+d_{k} \cos (k t)\right) \\
& =\frac{16}{3} \sum_{k=1}^{\infty}\left(1+\frac{9 / 4}{k^{2}-9 / 4}\right)\left(c_{k} \sin (k t)+d_{k} \cos (k t)\right)
\end{aligned}
$$

Clearly we have that if $p \in C[0,2 \pi]$, then $p \in L^{2}[0,2 \pi]$ and, by $(34), L(p(t)) \in$ $L^{2}[0,2 \pi]$. Now see us that $L(p(t))$ is continuous in $[0,2 \pi]$. Fom $(34)$ we have

$$
\begin{aligned}
L(p(t))= & \frac{16 \pi}{3} p(t)+\frac{16}{3} \sum_{k=1}^{\infty} \frac{9 / 4}{k^{2}-9 / 4} c_{k} \sin (k t) \\
& +\frac{16}{3} \sum_{k=1}^{\infty} \frac{9 / 4}{k^{2}-9 / 4} d_{k} \cos (k t) \\
\equiv & \frac{16}{3}\left(p(t)+S_{1}(t)+S_{2}(t)\right)
\end{aligned}
$$


Let now $t_{n} \rightarrow t$. Thus

$$
\begin{aligned}
\left|S_{1}\left(t_{n}\right)-S_{1}(t)\right| \leq & \sum_{k=1}^{\infty} \frac{9 / 4}{\left|k^{2}-9 / 4\right|}\left|c_{k}\right|\left|\sin \left(k t_{n}\right)-\sin (k t)\right| \\
& \leq \frac{9}{4} \sum_{k=1}^{\infty} \frac{k}{\left|k^{2}-9 / 4\right|}\left|c_{k}\right||\cos (\zeta)|\left|t_{n}-t\right| \\
& \leq 9 \sum_{k=1}^{\infty} \frac{1}{k}\left|c_{k}\right|\left|t_{n}-t\right| \\
& \leq 9\left(\sum_{k=1}^{\infty} \frac{1}{k^{2}}\right)^{1 / 2}\left(\sum_{k=1}^{\infty}\left(c_{k}\right)^{2}\right)^{1 / 2}\left|t_{n}-t\right| \\
& \leq c\left|t_{n}-t\right|,
\end{aligned}
$$

where the constant $c$ is independent of $t$ and $t_{n}$. Hence $S_{1}$ is a continuous function. Similarly, $S_{2}$ is also a continuous function. Hence, by (35) and (36), $L(p) \in C[0,2 \pi]$ if $p \in C[0,2 \pi]$.

Furthermore for all $k$ positive integer, $1+\frac{9 / 4}{k^{2}-9 / 4} \neq 0$ and

$$
\begin{aligned}
p(t) & =\sum_{k=1}^{\infty}\left(c_{k} \sin (k t)+d_{k} \cos (k t)\right) \\
& =\sum_{k=1}^{\infty}\left(1-\frac{9}{4 k^{2}}\right)\left(1+\frac{9 / 4}{k^{2}-9 / 4}\right)\left(c_{k} \sin (k t)+d_{k} \cos (k t)\right) .
\end{aligned}
$$

Therefore, following the arguments in (34), (35) and (36) we have that the operator $L^{-1}: C[0,2 \pi] \rightarrow C[0,2 \pi]$ defined by

$$
L^{-1}(q(t))=\frac{3}{16 \pi} \sum_{k=1}^{\infty}\left(1-\frac{9}{4 k^{2}}\right)\left(f_{k} \sin (k t)+g_{k} \cos (k t)\right)
$$

is the inverse of $L$. Here $\sum_{k=1}^{\infty}\left(f_{k} \sin (k t)+g_{k} \cos (k t)\right)$ is the Fourier series of $q(t)$. So, by Theorem 1 , there exists $\epsilon_{0}>0$ such that for $\epsilon \in\left(0, \epsilon_{0}\right)$ the equation ( 7$)$ has solution $u \in C^{0}(\Omega)$.

Lemma 1. If $h(t, x)=9 \sin (3 x)$ and $H=\square^{-1}(h)+v$, with $v \in N$, then $H$ changes sign.

Proof. By the definition of $H, H(t, x)=\sin 3 x+v(t, x)$ with $v(t, x)=p(t+x)-$ $p(t-x)$ (see (3)). Assuming that $H(t, x)>0$ for all $x \in(0, \pi), t \in[0,2 \pi]$, we have that $H(t, \pi / 2)=-1+p(t+\pi / 2)-p(t-\pi / 2)>0$. Hence

$$
\begin{aligned}
\int_{0}^{2 \pi} p(\pi / 2+t) d t & >\int_{0}^{2 \pi}(1+p(t-\pi / 2)) d t \\
& =2 \pi+\int_{0}^{2 \pi} p(t-\pi / 2) d t .
\end{aligned}
$$


Since $p$ is $2 \pi$-periodic and $\int_{0}^{2 \pi} p(t) d t=0,(39)$ is a contradiction. On the other hand, if we assume that $H(t, x)<0$ for all $x \in(0, \pi), t \in[0,2 \pi]$, taking $x=\pi / 6$ we also reach a contradiction. Hence $H$ changes sign.

Taking $h(t, x)=9 \sin (3 x)$ and $H(t, x)=\sin (3 x)$, by (38) and Theorem 1 , the equation (1) has a solution for $\epsilon$ small. On the other hand, by Lemma 1, Theorem 1 of [1] does not apply because neither $h$ nor $H$ are of one sign. These arguments easily extend to any function $h(t, x)$ of the form $\sin (k x)$ with $k$ odd and positive. This provides a large class of examples for which Theorem 1 applies but not Theorem 1 of $[1]$.

Since the set of invertible of operators in a Banach space is open in the algebra of such operators, if $H_{1}(t, x)$ is small then $L+L_{H_{1}}$ is also invertible. Thus we have:

Theorem 3. There exists $\delta>0$ such that if $\left\|h_{1}\right\| \leq \delta$ then there exists $\epsilon_{0}>0$ such that for $\epsilon \in\left(0, \epsilon_{0}\right), h(t, x)=9 \sin (3 x)+h_{1}(t, x) \in N^{\perp}$, and $k=1$ the equation (1) has a solution. Moreover, every solution to $\square H=h$ satisfying the boundary condition in (1) changes sign.

\section{Appendix}

The purpose of this appendix is to establish the existence of $\hat{v}=\epsilon V$ as used in the proof of the case $k>1$ in Theorem 1 when $H$ is positive (see Lemma 3 below.)

Lemma 2. There exists $\Delta$ such that for any $v \in N \cap L^{2 k}(\Omega)$

$$
\int_{\Omega} v^{2 k}(t, x) d \sigma \leq \Delta \int_{\Omega_{1}} v^{2 k}(t, x) d \sigma
$$

where $\Omega_{1}=\{(t, x) ;|x-\pi / 2| \leq \pi / 4, t \in[0,2 \pi]\}$.

Proof. For $v \in N \cap L^{2 k}(\Omega)$, let

$$
A=\left\{x \in[\pi / 4,3 \pi / 4] ; \int_{0}^{2 \pi} v^{2 k}(t, x) d t \geq \frac{20}{\pi} \int_{\Omega_{1}} v^{2 k}(t, x) d \sigma\right\} .
$$

By Fubini's Theorem $m(A) \leq \pi / 20$. Let $B=[\pi / 4,3 \pi / 4]-A$. Hence

$$
\begin{aligned}
m(B) & \geq 9 \pi / 20, \\
m([\pi / 4, \pi / 2] \cap B) & \geq m(B)-m([\pi / 2,3 \pi / 4]) \\
& \geq \pi / 5, \\
m([\pi / 2,3 \pi / 4] \cap B) & \geq \pi / 5 .
\end{aligned}
$$

Assuming that there is $x \in[0, \pi / 4)$ such that, for all $z \in B \cap[\pi / 2,3 \pi / 4], z-x \notin B$ we see that $B_{1}=\{z-x ; z \in B \cap[\pi / 2,3 \pi] / 4\} \subset A$. Hence $m(A) \geq m\left(B_{1}\right) \geq \pi / 5$ which contradicts that $m(A) \leq \pi / 20$. Thus for all $x \in[0, \pi / 4)$, there exists $z \in$ $B \cap[\pi / 2,3 \pi / 4]$ such that $z-x \in B$. Now for $t \in[0,2 \pi], x \in[0, \pi / 4)$ we have 


$$
\begin{aligned}
\left|v^{2 k}(t, x)\right| & =|v(t-z, z-x)+v(t-x, 0)-v(t+x-z, z)|^{2 k} \\
& \leq|v(t-z, z-x)-v(t-x-z, z)|^{2 k} \\
& \leq 2^{2 k-1}\left(v^{2 k}(t-z, z-x)+v^{2 k}(t-x-z, z)\right) .
\end{aligned}
$$

Thus

$$
\begin{aligned}
\int_{0}^{2 \pi} v^{2 k}(t, x) d t & \leq 2^{2 k-1}\left(\int_{0}^{2 \pi} v^{2 k}(t, z-x) d t+\int_{0}^{2 \pi} v^{2 k}(t, z) d t\right) \\
& \leq 2^{2 k} \frac{20}{\pi} \int_{\Omega_{1}} v^{2 k}(t, x) d \sigma .
\end{aligned}
$$

Similarly, for all $x \in(3 \pi / 4, \pi]$,

$$
\int_{0}^{2 \pi} v^{2 k}(t, x) d t \leq 2^{2 k} \frac{20}{\pi} \int_{\Omega_{1}} v^{2 k}(t, x) d \sigma .
$$

Consequently, by Fubini's theorem, (44), and (45)

$$
\begin{aligned}
\int_{\Omega} v^{2 k}(t, x) d \sigma= & \int_{0}^{\pi / 4} \int_{0}^{2 \pi} v^{2 k}(t, x) d t d x+\int_{\Omega_{1}} v^{2 k}(t, x) d \sigma \\
& +\int_{3 \pi / 4}^{\pi} \int_{0}^{2 \pi} v^{2 k}(t, x) d t d x \\
\leq & \left.\left(1+5 \cdot 2^{2 k+1}\right)\right) \int_{\Omega_{1}} v^{2 k}(t, x) d \sigma \\
\equiv & \Delta \int_{\Omega_{1}} v^{2 k}(t, x) d \sigma
\end{aligned}
$$

which proves Lemma (2).

Lemma 3. If $H$ is continuous and positive in $\mathbb{R} \times(0, \pi)$ then there exists $V \in N \cap L_{\infty}$ such that $\Pi_{N}(V+H)^{2 k}=0$.

Proof. Let

$$
\begin{aligned}
g(s, t, x) & =(s+H(t, x))^{2 k+1}-s^{2 k+1} \\
& =\sum_{j=1}^{2 k+1} \frac{(2 k+1) !}{(2 k+1-j) ! j !} s^{2 k+1-j} H^{j}(t, x) .
\end{aligned}
$$

As long as $H(t, x) \geq 0, g$ is a convex function of its first variable. Hence $f(v)=$ $\int_{\Omega} g(v(t, x), t, x) d \sigma$ defines a convex functional on $N \cap L^{2 k}(\Omega)$. By the continuity of of $H$, there exists a positive constant $C$ such that $H(t, x) \geq C$ for all $(t, x) \in \Omega_{1}$. This and Lemma 2 imply that $\lim _{\|v\|_{2 k} \rightarrow \infty} f(v)=+\infty$. Therefore there exists $V \in$ $N \cap L^{2 k}(\Omega)$ such that $f(V)=\min \left\{f(v) ; v \in N \cap L^{2 k}(\Omega)\right\}$ (see [10], Theorem 7.3.4).

Let us see that $V$ is in $L^{\infty}(\Omega)$. Let $p: \mathbb{R} \rightarrow \mathbb{R}$ be a $2 \pi$-periodic function such that $V(t, x)=p(t+x)-p(t-x)$. Let $\varphi$ be as in (15). Since $\varphi \in N \cap L^{2 k}(\Omega)$ and $V^{2 k} \in N^{\perp}, 0=\int_{\Omega} \varphi(V+H)^{2 k} d \sigma=\int_{\Omega} \varphi\left((V+H)^{2 k}-V^{2 k}\right) d \sigma$ (see Lemma 2.4 in [1]). 
Arguing as in (17)-(18) we have

$$
\begin{aligned}
0= & \int_{0}^{\pi}(V+H)^{2 k}(r+x, x) d x-\int_{0}^{\pi}(V+H)^{2 k}(r-x, x) d x \\
= & \int_{0}^{\pi} \sum_{j=1}^{2 k} \frac{(2 k) !}{(2 k+1-j) ! j !}(p(r+2 x)-p(r))^{2 k-j} H^{j}(r+x, x) d x \\
& -\int_{0}^{\pi} \sum_{j=1}^{2 k} \frac{(2 k) !}{(2 k+1-j) ! j !}(p(r)-p(r-2 x))^{2 k-j} H^{j}(r-x, x) d x \\
= & -2 k p^{2 k-1}(r)\left(\int_{0}^{\pi}(H(r+x, x)+H(r-x, x)) d x\right) \\
& +\sum_{j=2}^{2 k} p^{2 k-j}(r) q_{j}(r),
\end{aligned}
$$

where the $q_{j}$ 's are bounded periodic functions. Since also we are assuming $H$ to be continuous and positive, there exists a positive constant $c$ such that $\int_{0}^{\pi}(H(r+x, x)+$ $H(r-x, x)) d x \geq c$ for all $r \in[0,2 \pi]$. This and (48) imply that $p \in L^{\infty}(\mathbb{R})$. Hence $V \in L^{\infty}(\mathbb{R})$, which proves Lemma 3 .

\section{References}

[1] Berti, M. and Biasco, L. Forced vibrations of wave equations with non-monotone nonlinearities, Ann. Inst. H. Poincaré Anal. Non Linaire, 23 (2006), no. 4, 439-474.

[2] Brézis, H. and Nirenberg, L., Forced vibrations for a nonlinear wave equation, Comm. Pure Appl. Math., 31 (1978), 1-30.

[3] Brézis, H. and Nirenberg, L., Characterizations of the ranges of some nonlinear operators and applications to boundary value problems, Ann. Sc. Norm. Sup. di Pisa, (1978), 225326.

[4] Caicedo, J. F. and Castro, A. A semilinear wave equation with derivate of nonlinearity containing multiple eigenvalues of infinite multiplicity, Contemporary Math., 208 (1997),111-132.

[5] Caicedo, J. F. and Castro, A., A semilinear wave equation with smooth data and no resonance having no continuous solution, Disc. and Cont. Dynamical Systems, 24 (2009), 653-658.

[6] Castro, A. and Preskill B., Existence of solutions for a semilinear wave equation with non-monotone nonlinearity, Continuous and Discrete Dynamical Systems, Series A, Vol. 28, No. 2, (2010), 649-658.

[7] Castro, A. and Unsurangsie S., A semilinear wave equation with nonmonotone nonlinearity, Pacific Jour. of Math., 132 (1988), 215-225.

[8] Coron, J. M. Periodic solutions of a nonlinear wave equation without assumption of monotonicity, Math. Ann. 262 (1983), no. 2, 273285.

[9] Hofer, H., On the range of a wave operator with nonmonotone nonlinearity, Math. Nachr., 106 (1980), 327-340. 
[10] Kurdila, A. and Zabarankin, M., Convex functional analysis, Volume 1, Birkhauser.

[11] Lovicarová, H., Periodic solutions of a weakly wave equation in one dimension, Czech. Math. Jour., 19 (1969), 324-342.

[12] Rabinowitz, P.H., Large amplitude time periodic solutions of a semilinear wave equation, Comm. Pure Appl. Math. 37 (1984), no. 2, 189-206.

[13] Willem, M., Density of the range of potential operators, Proc. Amer. Math. Soc. 83 (1981), no. 2, 341-344.

José F. Caicedo

Departamento de Matemáticas

Universidad Nacional de Colombia

Bogotá

Colombia

e-mail: jfcaicedoc@unal.edu.co

Alfonso Castro

Department of Mathematics

Harvey Mudd College

Claremont, CA 91711

USA

e-mail: castro@math.hmc.edu

Rodrigo Duque

Departamento de Matemáticas

Universidad Nacional de Colombia

Bogotá

Colombia

e-mail: rduqueba@unal.edu.co

Received: December 12, 2010. 